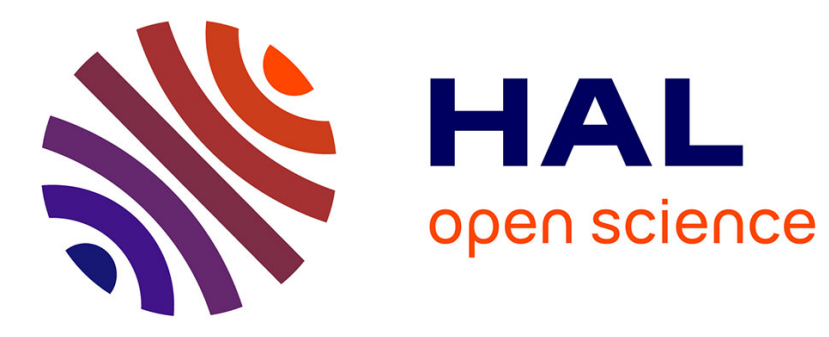

\title{
Auxiliary Signal Design for Robust Multi-Model Identification
}

Ramine Nikoukhah, Stephen L. Campbell, K.G. Horton, François Delebecque

\section{To cite this version:}

Ramine Nikoukhah, Stephen L. Campbell, K.G. Horton, François Delebecque. Auxiliary Signal Design for Robust Multi-Model Identification. [Research Report] RR-4000, INRIA. 2000. inria-00072644

\section{HAL Id: inria-00072644 \\ https://hal.inria.fr/inria-00072644}

Submitted on 24 May 2006

HAL is a multi-disciplinary open access archive for the deposit and dissemination of scientific research documents, whether they are published or not. The documents may come from teaching and research institutions in France or abroad, or from public or private research centers.
L'archive ouverte pluridisciplinaire HAL, est destinée au dépôt et à la diffusion de documents scientifiques de niveau recherche, publiés ou non, émanant des établissements d'enseignement et de recherche français ou étrangers, des laboratoires publics ou privés. 
INSTITUT NATIONAL DE RECHERCHE EN INFORMATIQUE ET EN AUTOMATIQUE

\title{
Auxiliary signal design for robust multi-model identification
}

\author{
R. Nikoukhah - S. L. Campbell - K.G. Horton ${ }^{\dagger}$ - F. Delebecque*
}

\section{$\mathbf{N}^{\circ} 4000$}

September 2000

THÈME 4 



\title{
Auxiliary signal design for robust multi-model identification
}

\author{
R. Nikoukhah*, S. L. Campbell ${ }^{\dagger}$, K.G. Horton ${ }^{\dagger}$, F. Delebecque* \\ Thème 4 -Simulation et optimisation \\ de systèmes complexes \\ Projet Metalau
}

Rapport de recherche $n^{\circ} 4000$ - September 2000 -26 pages

\begin{abstract}
Under the assumption that one of two given linear models is the real underlying model of the system, a proper auxiliary signal is defined as an input signal that allows the selection of the correct model. Under the assumption that model noises have bounded energy, the separability index is defined as the inverse of the energy of the proper auxiliary signal of least energy. A method for the computation of this index and the construction of the auxiliary signal is presented. Finally, an efficient procedure for on-line identification called the hyperplane test is developed.
\end{abstract}

Key-words: identification, Failure detection, Auxiliary signal design

* INRIA, Rocquencourt, BP 105, 78153 Le Chesnay cedex, France

$\dagger$ North Carolina State University, Raleigh, NC 27695-8205. Research supported in part by the National Science Foundation under DMS-9714811, DMS-9802259, and INT-9605114. 


\section{Calcul de signal de test pour l'identification robuste multi-modèle}

Résumé : Etant donnés deux modèles linéaires dont l'un représente le système réel, on calcule un signal de test qui permet la sélection du vrai modèle. Sous l'hypothèse que les bruits de modélisation sont d'énergie bornée, on cherche un signal de détection d'énergie minimale. L'inverse de l'énergie d'un tel signal est appelé l'indice de séparabilité. On donne une méthode de calcul de cet indice et la construction d'un signal de détection. Enfin on donne une procédure d'identification en ligne appelée "test de l'hyperplan".

Mots-clés : identification, détection de panne, signal de test 


\section{Introduction}

Multi-model identification means that we have two or more candidate models for a system, and based on the measurements of the inputs and the outputs of the system over a test period, we have to decide which model corresponds to the system. It is not always possible to exclude all models except for one in a reasonable way based on the input-output measurements unless the input signal has certain properties. An input signal designed specifically for multi-model identification over a test period is called an auxiliary signal or a detection signal. The latter terminology is used more in the failure detection context.

Even though the design of auxiliary signals has been an issue in the system identification literature, the point of view adopted here is closer to that found in failure detection problems, in particular $[12,5,6]$; see also [11]. However these works, and most problem formulations for auxiliary signal design in the identification literature, use statistical methods and consider model uncertainties as stochastic processes.

The work presented here is different in that uncertainties are modeled as bounded energy signals (such as in $H_{\infty}$ control theory, robust estimation and robust model validation, see for example [10]) and zero-error identification is envisaged. This type of problem formulation has been first considered in [9] for discrete time systems. The proposed solution there involved the recursive construction of a matrix with increasing size (something which is feasible only for short test periods) and could not be extended to the continuous-time case.

In this paper, we show that the auxiliary signal can be obtained as a solution of a boundary value system which can be efficiently solved using a "game-type" Riccati equation, even for long test periods. But most importantly, we show that the solution of this boundary value system can be used to solve the on-line identification problem in a very elegant and efficient way.

The fundamental assumptions in this paper are that we have two candidate models: Model 0 and Model 1. Each model is linear and subject to bounded energy noise. The finite-energy assumption on the noises allows us to consider zero-error identification. Consider the input (auxiliary signal) $v$ and let $\mathcal{A}^{0}(v)$ represent the set of possible outputs $y$ associated with this input, if Model 0 were the correct model. Similarly, let $\mathcal{A}^{1}(v)$ represent the set of outputs under the assumption that Model 1 is the correct model. Then clearly for perfect identification we need that

$$
\mathcal{A}^{0}(v) \cap \mathcal{A}^{1}(v)=\emptyset .
$$

This can be achieved thanks to the finite-energy assumption on the noises. The minimum energy required by $v$ to impose (1.1) is a measure of how distinct the two models are, and how easy it is to distinguish them. We call its inverse the separability index.

The construction of the separability index and the corresponding optimal auxiliary signal is done off-line, so it can be computationally extensive. The on-line identification on the other hand must require as little computation as possible. For that, we develop the hyperplane test which has been first introduced in [8] in a different context (different assumptions on the noises).

The outline of the paper is as follows. After problem formulation in Section 2, a method for computing the separability index is presented in Section 3. In Section 4, the optimal auxiliary signal $v$ is constructed. The on-line identification problem is considered in Section 5. An example and discussion are in Section 6. 


\section{Problem formulation}

\subsection{System models}

The true system model is supposed to be one of the following two models over the test period $[0, T]$ :

$$
\begin{aligned}
\dot{x}_{i} & =A_{i} x_{i}+B_{i} v+M_{i} \nu_{i}, \\
y & =C_{i} x_{i}+N_{i} \nu_{i}
\end{aligned}
$$

for $i=0$ and $1 . v$ is the auxiliary signal, $y$ is the output, $x_{i}$ 's are the states and $\nu_{i}$ 's represent perturbations, noises and unmeasured inputs. $A_{i}, B_{i}, C_{i}, M_{i}, N_{i}$ are matrices of appropriate dimensions. These matrices could be time varying but each entry must be a continuous function of time. Finally, we assume that the $N_{i}$ 's are surjective.

Note that the states of the two models $x_{0}$ and $x_{1}$ need not have the same dimensions. The same is true for $\nu_{0}$ and $\nu_{1}$. The only things that tie together the two models are $y$ and $v$.

The assumptions on the noises and the initial states are

$$
\mathcal{S}_{i}\left(x_{i}(0), \nu_{i}\right) \triangleq x_{i}(0)^{T} P_{i, 0}^{-1} x_{i}(0)+\int_{0}^{T}\left\|\nu_{i}\right\|^{2} d t<1, \quad i=0,1 .
$$

Note that $\mathcal{S}_{i}\left(x_{i}(0), \nu_{i}\right)$ measures the size of the disturbances that our model identification is to be robust to.

\subsection{Proper auxiliary signal}

We say that the $\mathcal{L}^{2}[0, T]$ vector function $v$ is a proper auxiliary signal if its application implies that we are able to always distinguish the two candidate models based on observation $y$. That is condition (1.1) is satisfied.

Defi nition 2.1 The auxiliary signal $v$ is not proper if there exist $x_{0}, x_{1}, \nu_{0}, \nu_{1}$, and $y$ satisfying (2.1), (2.2) and (2.3) both for $i=0$ and $i=1$. The auxiliary signal $v$ is called proper otherwise.

Proper auxiliary signals exist under quite weak conditions. Note that $\mathcal{A}^{0}(v)$ and $\mathcal{A}^{1}(v)$ are translates by $y_{0}$ and $y_{1}$ of bounded sets where $y_{0}$ and $y_{1}$ are respectively the outputs of Model 0 and Model 1 corresponding to zero noise and zero initial state. Since $y_{0}$ and $y_{1}$ depend linearly on $v$, either $y_{0}=y_{1}$ for all $v$, or $y_{0}-y_{1}$ can be made arbitrarily large with proper choice of $v$. So proper auxiliary signals exist provided the linear mapping from $v$ to $y_{0}$ is distinct from the linear mapping from $v$ to $y_{1}$. In the time invariant case, this is equivalent to

$$
C_{0}\left(s I-A_{0}\right)^{-1} B_{0}-C_{1}\left(s I-A_{1}\right)^{-1} B_{1} \neq 0 .
$$

Defi nition 2.2 Let $V$ denote the set of proper auxiliary signals $v$. Then,

$$
\gamma^{*}=\left(\inf _{v \in V} \int_{0}^{T}\|v\|^{2} d t\right)^{-1},
$$

is called the separability index associated with (2.1)-(2.2). 
Clearly, $\gamma^{*-1}$ is a lower bound on the energy of proper auxiliary signals. So, greater separability index implies existence of lower energy proper auxiliary signal. The separability index is zero when there is no proper auxiliary signal. Note that if a proper auxiliary signal exists, then the minimum norm is nonzero since the $N_{i}$ are surjective.

Auxiliary signal $v$ being not proper means that there exist $x_{0}, \nu_{0}, x_{1}, \nu_{1}$ and $y$ compatible with (2.1)-(2.2), $i=0,1$, such that

$$
\max \left\{\mathcal{S}_{0}\left(x_{0}(0), \nu_{0}\right), \mathcal{S}_{1}\left(x_{1}(0), \nu_{1}\right)\right\}<1
$$

where $\mathcal{S}_{i}$ are defined in (2.3). We can rewrite this inequality as follows

$$
\max _{0 \leq \beta \leq 1} L\left(\left(x_{0}, \nu_{0}, x_{1}, \nu_{1}\right), \beta\right)<1
$$

where

$$
L\left(\left(x_{0}, \nu_{0}, x_{1}, \nu_{1}\right), \beta\right)=\beta \mathcal{S}_{0}\left(x_{0}(0), \nu_{0}\right)+(1-\beta) \mathcal{S}_{1}\left(x_{1}(0), \nu_{1}\right) .
$$

We thus obtain the following characterization of properness.

Lemma 2.1 The auxiliary signal $v$ is not proper if and only if

$$
\inf \max _{0 \leq \beta \leq 1} L\left(\left(x_{0}, \nu_{0}, x_{1}, \nu_{1}\right), \beta\right)<1
$$

where the infimum is taken over $x_{i}, \nu_{i}$ and $y$ in $\mathcal{L}^{2}[0, T]$, subject to (2.1)-(2.2), $i=0,1$.

Note that the output $y$ can easily be eliminated from the constraints (2.1)-(2.2), $i=0,1$, by subtracting (2.2) for $i=0$ from (2.2) for $i=1$, giving

$$
0=C_{0} x_{0}-C_{1} x_{1}+N_{0} \nu_{0}-N_{1} \nu_{1}
$$

We thus end up with an infimum taken over $S_{v}$, the set of $\mathcal{L}^{2}[0, T]$ functions $\left(x_{0}, \nu_{0}, x_{1}, \nu_{1}\right)$ satisfying the constraints (2.1), $i=0,1$, and (2.10).

Let us now define the following function which will prove very useful later on,

$$
J_{v}(\beta)=\inf _{\left(x_{0}, \nu_{0}, x_{1}, \nu_{1}\right) \in S_{v}} L\left(\left(x_{0}, \nu_{0}, x_{1}, \nu_{1}\right), \beta\right)
$$

Lemma 2.2 For all $v \in \mathcal{L}^{2}[0, T]$, for $0 \leq \beta \leq 1, J_{v}(\beta)$ is defined and has the following properties:

1. it is zero for $\beta=0$ and $\beta=1$,

2. it is quadratic in $v$, i.e., for all scalar $c, J_{c v}(\beta)=|c|^{2} J_{v}(\beta)$.

3. it is a continuous and concave function of $\beta$, 
Proof Let $v$ be any $\mathcal{L}^{2}[0, T]$ function. The set $S_{v}$ is not empty. This follows from the surjectivity of the $N_{i}$ 's. In fact, for any choice of $x_{0}(0)$ and $\nu_{0} \in \mathcal{L}^{2}[0, T]$, there exist $x_{1}$ and $\nu_{1}$ such that $\left(x_{0}, \nu_{0}, x_{1}, \nu_{1}\right) \in S_{v}$, and vice-versa.

Since $S_{v}$ is not empty and $L\left(\left(x_{0}, \nu_{0}, x_{1}, \nu_{1}\right), \beta\right) \geq 0$, we conclude that $J_{v}(\beta)$ is defined.

To show part 1, let $\bar{x}_{0}(0)=0$ and $\bar{\nu}_{0}=0$. Then there exists $\left(\bar{x}_{1}, \bar{\nu}_{1}\right)$ such that $\left(\bar{x}_{0}, \bar{\nu}_{0}, \bar{x}_{1}, \bar{\nu}_{1}\right) \in$ $S_{v}$. Thus,

$$
J_{v}(\beta) \leq L\left(\left(\bar{x}_{0}, \bar{\nu}_{0}, \bar{x}_{1}, \bar{\nu}_{1}\right), \beta\right)=(1-\beta) \mathcal{S}_{1}\left(\bar{x}_{1}(0), \bar{\nu}_{1}\right)
$$

so for $\beta=1$, we get $J_{v}(1)=0$. Similarly we can show that $J_{v}(0)=0$.

Part 2 is straightforward. Simply note that $J_{v}(\beta)$ is a quadratic cost function subject to linear constraints.

For Part 3, first we show that $J_{v}(\beta)$ is concave. Let $0 \leq \alpha \leq 1$, and $0 \leq \beta_{1}<\beta_{2} \leq 1$. Then

$$
\begin{gathered}
\alpha J_{v}\left(\beta_{1}\right)+(1-\alpha) J_{v}\left(\beta_{2}\right)= \\
\alpha \inf \left\{\beta_{1} \mathcal{S}_{0}+\left(1-\beta_{1}\right) \mathcal{S}_{1}\right\}+(1-\alpha) \inf \left\{\beta_{2} \mathcal{S}_{0}+\left(1-\beta_{2}\right) \mathcal{S}_{1}\right\} \leq \\
\inf \left\{\left(\alpha \beta_{1}+(1-\alpha) \beta_{2}\right) \mathcal{S}_{0}+\left(\alpha\left(1-\beta_{1}\right)+(1-\alpha)\left(1-\beta_{2}\right)\right) \mathcal{S}_{1}\right\}= \\
J_{v}\left(\alpha \beta_{1}+(1-\alpha) \beta_{2}\right)
\end{gathered}
$$

which shows that $J_{v}(\beta)$ is concave which in turn implies that it is continuous, except possibly at the end-point $\beta=0,1$. Again consider $\left(\bar{x}_{0}, \bar{\nu}_{0}, \bar{x}_{1}, \bar{\nu}_{1}\right)$ defined above and (2.12). As $\beta$ goes to one, the right hand side of this equation goes to zero, thus so does $J_{v}(\beta)$. Thus $J_{v}(\beta)$ is continuous at one. Similarly, we can show that it is continuous at zero.

It turns out that the optimization problem (2.11) corresponds to a well studied problem in the literature. Just interpret $\nu_{0}$ and $\nu_{1}$ as zero-mean white Gaussian noise processes with covariance $I / \beta$ and $I /(1-\beta)$ respectively and consider $x_{0}(0)$ and $x_{1}(0)$ to be independent Gaussian vectors with covariance $P_{0,0} / \beta$ and $P_{1,0} /(1-\beta)$. Then $L\left(\left(x_{0}, \nu_{0}, x_{1}, \nu_{1}\right), \beta\right)$ is nothing but the negative of the $\log$-likelihood function corresponding to System (2.1), $i=0,1$. Minimizing $L\left(\left(x_{0}, \nu_{0}, x_{1}, \nu_{1}\right), \beta\right)$ subject to the observation equation (2.10) gives the smoothed estimate of $\left(x_{0}, \nu_{0}, x_{1}, \nu_{1}\right)$. Note that here, the observation happens to be identically zero.

The solution of this optimization problem can be obtained by solving the fixed interval smoother problem (see for example Chapter 5 in [4]). We thus obtain the following result.

Lemma 2.3 For all $v \in \mathcal{L}^{2}[0, T]$, the infimum is attained in (2.11), and if $0<\beta<1$, the minimum is unique and continuous in $\beta$. 
Theorem 2.1 The function $L$ has at least one saddle point $\left(\left(x_{0}^{s}, \nu_{0}^{s}, x_{1}^{s}, \nu_{1}^{s}\right), \beta^{s}\right)$ on $S_{v} \times[0,1]$ and

$$
\begin{aligned}
\inf _{\left(x_{0}, \nu_{0}, x_{1}, \nu_{1}\right) \in S_{v}} \max _{\beta \in[0,1]} L\left(\left(x_{0}, \nu_{0}, x_{1}, \nu_{1}\right), \beta\right)= & \\
& \min _{\left(x_{0}, \nu_{0}, x_{1}, \nu_{1}\right) \in S_{v}} \max _{\beta \in[0,1]} L\left(\left(x_{0}, \nu_{0}, x_{1}, \nu_{1}\right), \beta\right)= \\
\max _{\beta \in[0,1]} \min _{\left(x_{0}, \nu_{0}, x_{1}, \nu_{1}\right) \in S_{v}} L\left(\left(x_{0}, \nu_{0}, x_{1}, \nu_{1}\right), \beta\right)= & L\left(\left(x_{0}^{s}, \nu_{0}^{s}, x_{1}^{s}, \nu_{1}^{s}\right), \beta^{s}\right) .
\end{aligned}
$$

Proof Let $\left(\bar{x}_{0}^{\beta}, \bar{\nu}_{0}^{\beta},{\overline{x_{1}}}^{\beta}, \bar{\nu}_{1}^{\beta}\right)$ denote the solution of the problem (2.11). Then $\mathcal{S}_{i}\left(\bar{x}_{i}^{\beta}(0), \bar{\nu}_{i}^{\beta}\right), i=$ 0,1 , depend continuously on $0<\beta<1$. Moreover, since

$$
\mathcal{S}_{0}\left(\bar{x}_{0}^{\beta}(0), \bar{\nu}_{0}^{\beta}\right)=0, \quad \text { if } \beta=1,
$$

$\mathcal{S}_{0}\left(\bar{x}_{0}^{\beta}(0), \bar{\nu}_{0}^{\beta}\right)$ is continuous for $\beta \in(0,1]$, and since

$$
\mathcal{S}_{1}\left(\bar{x}_{1}^{\beta}(0), \bar{\nu}_{1}^{\beta}\right)=0, \quad \text { if } \beta=0,
$$

$\mathcal{S}_{1}\left(\bar{x}_{1}^{\beta}(0), \bar{\nu}_{1}^{\beta}\right)$ is continuous for $\beta \in[0,1)$.

Suppose

$$
\lim _{\beta \rightarrow 1} \mathcal{S}_{1}\left(\bar{x}_{1}^{\beta}(0), \bar{\nu}_{1}^{\beta}\right)>0 .
$$

Then for some $0<\beta^{s}<1$, we must have

$$
\mathcal{S}_{0}\left(\bar{x}_{0}^{\beta^{s}}(0), \bar{\nu}_{0}^{\beta^{s}}\right)=\mathcal{S}_{1}\left(\bar{x}_{1}^{\beta^{s}}(0), \bar{\nu}_{1}^{\beta^{s}}\right) .
$$

Let $\left(x_{0}^{s}, \nu_{0}^{s}, x_{1}^{s}, \nu_{1}^{s}\right)=\left(\bar{x}_{0}^{\beta^{s}}, \bar{\nu}_{0}^{\beta^{s}},{\overline{x_{1}}}^{\beta^{s}}, \bar{\nu}_{1}^{\beta^{s}}\right)$. Then

$$
L\left(\left(x_{0}^{s}, \nu_{0}^{s}, x_{1}^{s}, \nu_{1}^{s}\right), \beta\right) \leq \mathcal{S}_{1}\left(x_{1}^{s}(0), \nu_{1}^{s}\right), \quad \forall \beta \in[0,1],
$$

(in fact it is equal because $\beta$ cancels out) and

$$
L\left(\left(x_{0}, \nu_{0}, x_{1}, \nu_{1}\right), \beta^{s}\right) \geq \mathcal{S}_{1}\left(x_{1}^{s}(0), \nu_{1}^{s}\right), \quad \forall\left(x_{0}, \nu_{0}, x_{1}, \nu_{1}\right) \in S_{v}
$$

because $\left(x_{0}^{s}, \nu_{0}^{s}, x_{1}^{s}, \nu_{1}^{s}\right)$ is the solution of (2.11) for $\beta=\beta^{s}$. This implies that $\left(\left(x_{0}^{s}, \nu_{0}^{s}, x_{1}^{s}, \nu_{1}^{s}\right), \beta^{s}\right)$ is a saddle point and the rest follows (see for example Chapter VI, Section 1 of [3]).

Now suppose that (2.17) does not hold so that

$$
\lim _{\beta \rightarrow 1} \mathcal{S}_{1}\left(\bar{x}_{1}^{\beta}(0), \bar{\nu}_{1}^{\beta}\right)=0 .
$$

In that case $\mathcal{S}_{0}$ and $\mathcal{S}_{1}$ can be made arbitrarily small simultaneously. This implies that $J_{v}(\beta)=0$ for all $\beta$ which means that there exists $\left(x_{0}^{s}, \nu_{0}^{s}, x_{1}^{s}, \nu_{1}^{s}\right)$ such that (2.18) holds with equality to zero. Then, clearly (2.19) holds because both sides of the inequality are zero. And, (2.20) holds for all $\beta_{s} \in[0,1]$ because the right hand side of the inequality is zero and the left hand side cannot be negative. This implies that $\left(\left(x_{0}^{s}, \nu_{0}^{s}, x_{1}^{s}, \nu_{1}^{s}\right), \beta^{s}\right)$ is a saddle point and the rest follows.

RR $n^{\circ} 4000$ 
Corollary 2.1 Let $\left(\left(x_{0}^{s}, \nu_{0}^{s}, x_{1}^{s}, \nu_{1}^{s}\right), \beta^{s}\right)$ be a saddle point of $L$ on $S_{v} \times[0,1]$. If $\beta^{s} \neq 0$ and $\beta^{s} \neq 1$, then

$$
\mathcal{S}_{0}\left(x_{0}^{s}(0), \nu_{0}^{s}\right)=\mathcal{S}_{1}\left(x_{1}^{s}(0), \nu_{1}^{s}\right)
$$

Proof If $\left(\left(x_{0}^{s}, \nu_{0}^{s}, x_{1}^{s}, \nu_{1}^{s}\right), \beta^{s}\right)$ is a saddle point, then

$$
\max _{0 \leq \beta \leq 1} L\left(\left(x_{0}^{s}, \nu_{0}^{s}, x_{1}^{s}, \nu_{1}^{s}\right), \beta\right)=L\left(\left(x_{0}^{s}, \nu_{0}^{s}, x_{1}^{s}, \nu_{1}^{s}\right), \beta^{s}\right) .
$$

For notational convenience let $\mathcal{S}_{i}^{s}=\mathcal{S}_{i}\left(x_{i}^{s}(0), \nu_{i}^{s}\right)$. Then, we get that

$$
\max \left\{\mathcal{S}_{0}^{s}, \mathcal{S}_{1}^{s}\right\}=\beta^{s} \mathcal{S}_{0}^{s}+\left(1-\beta^{s}\right) \mathcal{S}_{1}^{s} .
$$

If we assume that $\mathcal{S}_{1}^{s} \geq \mathcal{S}_{0}^{s}$, we get $\mathcal{S}_{1}^{s}=\beta^{s} \mathcal{S}_{0}^{s}+\left(1-\beta^{s}\right) \mathcal{S}_{1}^{s}$ and conclude that $\mathcal{S}_{1}^{s}=\mathcal{S}_{0}^{s}$. Similarly, if we assume that $\mathcal{S}_{0}^{s} \geq \mathcal{S}_{1}^{s}$, we conclude that $\mathcal{S}_{1}^{s}=\mathcal{S}_{0}^{s}$.

Our original problem which consisted in finding a minimum energy proper auxiliary signal $v$ can now be expressed as follows:

$$
\inf \|v\| \text { subject to } \max _{0<\beta<1} J_{v}(\beta) \geq 1 \text {. }
$$

Note that we have excluded the cases $\beta=0$ and $\beta=1$ because $J_{v}(0)=J_{v}(1)=0$ as shown in Lemma 2.2.

Using the fact that $J_{v}(\beta)$ is quadratic in $v$ (Lemma 2.2), we obtain the following fundamental result:

Theorem 2.2 Let

$$
J^{*}(\beta)=\sup _{v \neq 0} \frac{J_{v}(\beta)}{\int_{0}^{T}\|v\|^{2} d t}
$$

Then

$$
\gamma^{* 2}=\max _{0<\beta<1} J^{*}(\beta)
$$

where $\gamma^{*}$ is the separability index defined previously.

Note that the larger $\gamma^{*}$ is, the easier it is to separate the two models. And when $\gamma^{*}=0$, then the two models are indistinguishable no matter what the input $v$ is. So, $\gamma^{*}$ can be considered as the deterministic counterpart of the Kullback distance [7] between two systems used in some stochastic formulations. Another interpretation of $\gamma^{*}$ is in terms of signal to noise ratio: $1 / \gamma^{*}$ can be seen as the smallest signal to noise ratio required for perfect identification. 


\section{Computation of the separability index}

We have seen in Theorem 2.2 that the separability index can be computed if we can compute $J_{v}(\beta)$ and $J^{*}(\beta)$.

To simplify the notations, let

$$
\begin{gathered}
x=\left(\begin{array}{l}
x_{0} \\
x_{1}
\end{array}\right), \nu=\left(\begin{array}{l}
\nu_{0} \\
\nu_{1}
\end{array}\right), \\
A=\left(\begin{array}{cc}
A_{0} & 0 \\
0 & A_{1}
\end{array}\right), M=\left(\begin{array}{cc}
M_{0} & 0 \\
0 & M_{1}
\end{array}\right), N=\left(\begin{array}{ll}
N_{0} & -N_{1}
\end{array}\right), \\
C=\left(\begin{array}{ll}
C_{0} & -C_{1}
\end{array}\right), B=\left(\begin{array}{c}
B_{0} \\
B_{1}
\end{array}\right), \\
P_{\beta}^{-1}=\left(\begin{array}{cc}
\beta P_{0,0}^{-1} & 0 \\
0 & (1-\beta) P_{1,0}^{-1}
\end{array}\right), V_{\beta}=\left(\begin{array}{cc}
\beta I & 0 \\
0 & (1-\beta) I
\end{array}\right) .
\end{gathered}
$$

Then the constraints (2.1), $i=0,1$, and (2.10) can be expressed as

$$
\begin{aligned}
\dot{x} & =A x+B v+M \nu \\
0 & =C x+N \nu
\end{aligned}
$$

and the function $J_{v}(\beta)$ becomes

$$
J_{v}(\beta)=\min _{(x, \nu) \in S_{v}} L((x, \nu), \beta)
$$

where (with slight abuse of notation)

$$
S_{v}=\left\{(x, \nu) \in \mathcal{L}^{2}[0, T] \times \mathcal{L}^{2}[0, T] \mid \text { (3.2) and (3.3) hold }\right\}
$$

and

$$
L((x, \nu), \beta)=x(0)^{T} P_{\beta}^{-1} x(0)+\int_{0}^{T} \nu^{T} V_{\beta} \nu d t
$$

\subsection{Computation of $J_{v}(\beta)$}

With this notation $J_{v}(\beta)$ is:

$$
J_{v}(\beta)=\min _{(x, \nu)}\left\{x(0)^{T} P_{\beta}^{-1} x(0)+\int_{0}^{T} \nu^{T} V_{\beta} \nu d t\right\}
$$

subject to (3.2)-(3.3). As noted earlier, this optimization problem corresponds exactly to a loglikelihood computation which can be solved by a Kalman filter for System (3.2)-(3.3) (simply interpret $\nu$ as a zero-mean white process with covariance $V_{\beta}^{-1}$ and $P_{\beta}$ as the initial error covariance; the observation happens to be identically zero).

$\mathrm{RR} \mathrm{n}^{\circ} 4000$ 
Lemma 3.1 Let

$$
\left(\begin{array}{cc}
Q_{\beta} & S_{\beta} \\
S_{\beta}^{T} & R_{\beta}
\end{array}\right)=\left(\begin{array}{c}
M \\
N
\end{array}\right) V_{\beta}^{-1}\left(\begin{array}{c}
M \\
N
\end{array}\right)^{T}
$$

Then the solution to the optimization problem (3.7) is given by

$$
J_{v}(\beta)=\int_{0}^{T} \hat{x}^{T} C^{T} R_{\beta}^{-1} C \hat{x} d t
$$

where $\hat{x}$ satisfies the "Kalman filter" equation

$$
\dot{\hat{x}}=\left(A-S_{\beta} R_{\beta}^{-1} C-P C^{T} R_{\beta}^{-1} C\right) \hat{x}+B v, \quad \hat{x}(0)=0
$$

and where $P$ is given by the Riccati equation

$$
\begin{aligned}
\dot{P}=\left(A-S_{\beta} R_{\beta}^{-1} C\right) P+P\left(A-S_{\beta} R_{\beta}^{-1} C\right)^{T}-P C^{T} R_{\beta}^{-1} C P+ & \\
& Q_{\beta}-S_{\beta} R_{\beta}^{-1} S_{\beta}^{T}, \quad P(0)=P_{\beta} .
\end{aligned}
$$

This is standard Kalman filter theory which can be found in many classical textbooks so the proof is omitted (see for example [4]).

\subsection{Computation of $J^{*}(\beta)$}

The following characterization of $J^{*}(\beta)$ as defined in (2.26), follows (3.9) and (3.10).

Lemma 3.2 Consider the following system

$$
\begin{aligned}
\dot{\hat{x}} & =\bar{A} \hat{x}+B v, \quad \hat{x}(0)=0 \\
z & =\bar{C} \hat{x}
\end{aligned}
$$

on $[0, T]$, where

$$
\begin{aligned}
\bar{A} & =A-S_{\beta} R_{\beta}^{-1} C-P C^{T} R_{\beta}^{-1} C \\
\bar{C} & =R_{\beta}^{-\frac{1}{2}} C .
\end{aligned}
$$

Then,

$$
J^{*}(\beta)=\sup _{v} \frac{\int_{0}^{T}\|z\|^{2} d t}{\int_{0}^{T}\|v\|^{2} d t} .
$$

This characterization allows us to develop a method for constructing the auxiliary signal $v$. 
Lemma 3.3 Consider the two-point boundary value system

$$
\left(\begin{array}{l}
\dot{\bar{x}} \\
\dot{\lambda}
\end{array}\right)=\left(\begin{array}{cc}
\bar{A} & -B B^{T} / \gamma^{2} \\
\bar{C}^{T} \bar{C} & -\bar{A}^{T}
\end{array}\right)\left(\begin{array}{l}
\bar{x} \\
\lambda
\end{array}\right)
$$

with $\bar{x}(0)=\lambda(T)=0$. Then, $J^{*}(\beta)$ is the largest value of $\gamma$ for which this boundary value system has non-trivial solution $(\bar{x}, \lambda)$. Moreover,

$$
v=-\frac{1}{J^{*}(\beta)} B^{T} \lambda
$$

maximizes (3.16).

Proof Suppose there exists a $v$ maximizing (3.16). Then this $v$ maximizes also

$$
\Gamma(v, \beta)=\int_{0}^{T}\|z\|^{2} d t-J^{*}(\beta) \int_{0}^{T}\|v\|^{2} d t .
$$

Then (3.17) and (3.18) follow from a direct application of the maximum principle.

On the other hand, suppose $(\bar{x}, \lambda)$ is a non-zero solution of (3.17) for some $\gamma$. Let $\bar{v}=-\frac{1}{\gamma} B^{T} \lambda$. Then $\bar{v} \neq 0$ because if $B^{T} \lambda=0$, then $\bar{x}=0$ which implies that $\lambda=0$. Then

$$
\begin{aligned}
\int_{0}^{T}\|\bar{v}\|^{2} d t & =\frac{1}{\gamma} \int_{0}^{T}-\bar{v}^{T} B^{T} \lambda d t \\
& =\frac{1}{\gamma} \int_{0}^{T}-(\dot{\bar{x}}-\bar{A} \bar{x})^{T} \lambda d t \\
& =\frac{1}{\gamma} \int_{0}^{T} \bar{x}^{T}\left(\dot{\lambda}+\bar{A}^{T} \lambda\right) d t \quad \text { (integration by parts) } \\
& =\frac{1}{\gamma} \int_{0}^{T} \bar{x}^{T} \bar{C}^{T} \bar{C} \bar{x} d t=\frac{1}{\gamma} \int_{0}^{T}\|z\|^{2} d t .
\end{aligned}
$$

Thus $\gamma \leq J^{*}(\beta)$ which implies $J^{*}(\beta)$ is the largest value of $\gamma$.

Lemma 3.3 does not provide a direct method for computing $J^{*}(\beta)$. The following result provides a more direct characterization of it.

Theorem 3.1 The Riccati equation

$$
\dot{\bar{P}}=\bar{A} \bar{P}+\bar{P} \bar{A}^{T}+\bar{P} \bar{C}^{T} \bar{C} \bar{P}+B B^{T} / \gamma^{2}, \quad \bar{P}(0)=0,
$$

has a solution on $[0, T]$ if and only if $\gamma^{2}>J^{*}(\beta)$.

Before giving the proof, we give the following useful result:

Lemma 3.4 Suppose a solution $\bar{P}$ to (3.21) exists on $[0, T]$. Then $\bar{P}(t)$ is positive semi-definite on $[0, T]$. Let $\bar{P}_{\gamma_{1}}$ and $\bar{P}_{\gamma_{2}}$ denote respectively the solutions of (3.21) for $\gamma=\gamma_{1}$ and $\gamma=\gamma_{2}$. If $\gamma_{1} \geq \gamma_{2}$, then $\bar{P}_{\gamma_{2}} \geq \bar{P}_{\gamma_{1}}$.

$\mathrm{RR} \mathrm{n}^{\circ} 4000$ 
Proof Let

$$
Q=\bar{P} \bar{C}^{T} \bar{C} \bar{P}+B B^{T} / \gamma^{2}
$$

Then (3.21) can be expressed as follows.

$$
\dot{\bar{P}}=\bar{A} \bar{P}+\bar{P} \bar{A}^{T}+Q, \quad \bar{P}(0)=0,
$$

But $Q$ is positive semi-definite so (3.23) is a Lyapunov equation. Thus $\bar{P}$ is positive semi-definite.

Now suppose $\gamma_{1} \geq \gamma_{2}$ and let $\bar{P}_{i}$ denote the solution of (3.23) corresponding to $\gamma=\gamma_{i}$ for $i=1,2$. Then by subtracting (3.23) for $i=1$ from (3.23) for $i=2$, we obtain

$$
\dot{\tilde{P}}=\left(\bar{A}+\bar{P}_{1} \bar{C}^{T} \bar{C}\right) \tilde{P}+\tilde{P}\left(\bar{A}+\bar{P}_{1} \bar{C}^{T} \bar{C}\right)^{T}+\tilde{P} \bar{C}^{T} \bar{C} \tilde{P}+\tilde{Q}, \quad \tilde{P}(0)=0,
$$

where $\tilde{P}=\bar{P}_{2}-\bar{P}_{1}$ and $\tilde{Q}=B B^{T} / \gamma_{2}^{2}-B B^{T} / \gamma_{1}^{2}$. But $\tilde{Q}$ is positive semi-definite, and (3.24) is again a Lyapunov equation. Thus $\tilde{P}$ is positive semi-definite which means that $\bar{P}_{\gamma_{2}} \geq \bar{P}_{\gamma_{1}}$.

Proof of Theorem 3.1 Let $\gamma$ satisfy

$$
\gamma^{2}>J^{*}(\beta)
$$

Then thanks to Lemma 3.3, the two-point boundary value system (3.17) has only zero solution. This is equivalent to $\Psi_{22}(0, T)$ being invertible where

$$
\Psi \triangleq\left(\begin{array}{ll}
\Psi_{11} & \Psi_{12} \\
\Psi_{21} & \Psi_{22}
\end{array}\right)
$$

is the state transition matrix associated with (3.17), i.e.,

$$
\frac{d}{d t}\left(\begin{array}{ll}
\Psi_{11} & \Psi_{12} \\
\Psi_{21} & \Psi_{22}
\end{array}\right)=\left(\begin{array}{cc}
\bar{A} & -B B^{T} / \gamma^{2} \\
\bar{C}^{T} \bar{C} & -\bar{A}^{T}
\end{array}\right)\left(\begin{array}{ll}
\Psi_{11} & \Psi_{12} \\
\Psi_{21} & \Psi_{22}
\end{array}\right)
$$

with $\Psi(0,0)=I$.

To pursue this proof, we need the following result.

Lemma 3.5 If $\gamma^{2}>J^{*}(\beta)$, then $\Psi_{22}(0, t)$ is invertible for all $0 \leq t \leq T$.

Proof Suppose there exists $t^{*}, 0<t^{*}<T$, such that $\Psi_{22}\left(0, t^{*}\right)$ is not invertible. Then, the two-point boundary value system (3.17) on $\left[0, t^{*}\right]$ with boundary condition $\bar{x}(0)=\lambda\left(t^{*}\right)=0$ has non-trivial solution $\left(\bar{x}_{*}, \lambda_{*}\right)$. On $\left[0, t^{*}\right]$, let

$$
v_{*}=-B^{T} \lambda_{*} / \gamma^{2}
$$


and let $v=v_{*}$ in (3.12)-(3.13) and denote the corresponding $z$ by $z_{*}$. Then we obtain

$$
\gamma^{2}=\frac{\int_{0}^{t^{*}}\left\|z_{*}\right\|^{2} d t}{\int_{0}^{t^{*}}\left\|v_{*}\right\|^{2} d t}
$$

Now let

$$
v(t)= \begin{cases}v_{*}(t) & \text { for } 0 \leq t \leq t^{*} \\ 0 & \text { for } t^{*}<t \leq T\end{cases}
$$

It is then clear that the output of (3.12)-(3.13) on $[0, T]$ corresponding to this $v$ satisfies $z(t)=z_{*}(t)$, for $t \leq t^{*}$ which implies that

$$
\frac{\int_{0}^{T}\|z\|^{2} d t}{\int_{0}^{T}\|v\|^{2} d t} \geq \frac{\int_{0}^{t^{*}}\left\|z_{*}\right\|^{2} d t}{\int_{0}^{t^{*}}\left\|v_{*}\right\|^{2} d t}=\gamma^{2} .
$$

But this contradicts (3.25) and (3.16). So $\Psi_{22}(0, t)$ is invertible.

Continuing with the proof of Theorem 3.1, let

$$
\bar{P}(t)=-\Psi_{12}(0, t) \Psi_{22}^{-1}(0, t) .
$$

Then from (3.27) follows (3.21) proving the existence of a solution to (3.21) over $[0, T]$ when $\gamma^{2}>$ $J^{*}(\beta)$.

Now we have to show that (3.21) does not have a solution over $[0, T]$ for any $\gamma$ such that $\gamma^{2} \leq$ $J^{*}(\beta)$. For that, we need first the following result.

Lemma 3.6 For $\gamma^{2}=J^{*}(\beta)$, the Riccati equation (3.21) has finite escape time $t^{*} \leq T$. That is, for some $0<t^{*} \leq T$, as $t$ goes to $t^{*}$, at least one of the eigenvalues of $\bar{P}(t)$ goes to infinity.

Proof Let $\gamma^{2}=J^{*}(\beta)$. Then (3.32) still defines the solution of (3.21) as long as $\Psi_{22}(0, t)$ is invertible. From Lemma 3.3 we have that (3.17) has a nontrivial solution so that $\Psi_{22}(0, T)$ is singular. Let $t^{*}$ be the first value of $t$ for which it is singular.

Suppose $\bar{P}\left(t^{*}\right)$ exists, then thanks to (3.32), it must satisfy

$$
\bar{P}\left(t^{*}\right) \Psi_{22}\left(0, t^{*}\right)=-\Psi_{12}\left(0, t^{*}\right)
$$

But $\Psi$ is a state transition matrix so it is invertible. Thus

$$
\Psi_{2}\left(0, t^{*}\right)=\left(\begin{array}{l}
\Psi_{12}\left(0, t^{*}\right) \\
\Psi_{22}\left(0, t^{*}\right)
\end{array}\right)
$$

is injective. But the matrix $\Psi_{22}\left(0, t^{*}\right)$ is singular which means that there exists a vector $w$ for which $\Psi_{22}\left(0, t^{*}\right) w=0$. Multiplying the two sides of (3.33) by $w$ on the right yields $\Psi_{12}\left(0, t^{*}\right) w=0$ which contradicts the injectivity of $\Psi_{2}\left(0, t^{*}\right)$. So as $t$ goes to $t^{*}, \bar{P}(t)$ diverges which thanks to Lemma 3.4 implies that at least one of its eigenvalues goes to infinity.

$\mathrm{RR} \mathrm{n}^{\circ} 4000$ 
To complete the proof of Theorem 3.1, we need to show that (3.21) does not have a solution over $[0, T]$ for any $\gamma$ such that $\gamma^{2} \leq J^{*}(\beta)$. Let $\bar{P}_{\bar{\gamma}}$, the solution of (3.21) for $\gamma=\bar{\gamma}$, exist on $[0, T]$ with $\bar{\gamma}^{2} \leq J^{*}(\beta)$. Thanks to Lemma 3.4, $\bar{P}_{\bar{\gamma}} \geq \bar{P}$ where $\bar{P}$ is the solution of (3.21) when $\gamma^{2}=J^{*}(\beta)$. But this is a contradiction because one of the eigenvalues of $\bar{P}(t)$ goes to infinity on $[0, T]$, so $\bar{P}_{\bar{\gamma}}$ cannot exist on $[0, T]$. This completes the proof of Theorem 3.1.

Theorem 3.1 allows us to compute $J^{*}(\beta)$ by a simple " $\gamma$-iteration" procedure, for example using a bisection method. The inconvenience is that we need to integrate two Riccati equations on $[0, T]$ for every iteration because $\bar{A}$ depends on $P$.

Theorem 3.2 The Riccati equation (3.21) has a solution (and thus $\gamma^{2}>J^{*}(\beta)$ ) if and only if the Riccati equation

$$
\begin{aligned}
\dot{\Pi}=\left(A-S_{\beta} R_{\beta}^{-1} C\right) \Pi+\Pi\left(A-S_{\beta} R_{\beta}^{-1} C\right)^{T}-\Pi C^{T} R_{\beta}^{-1} C \Pi+ \\
Q_{\beta}-S_{\beta} R_{\beta}^{-1} S_{\beta}^{T}-B B^{T} / \gamma^{2}, \Pi(0)=P_{\beta}
\end{aligned}
$$

has a solution on $[0, T]$.

Proof Let $\Pi=P-\bar{P}$. Then by subtracting (3.21) from (3.11), after some straightforward algebra, we obtain (3.35). The Riccati equation (3.11) does not depend on $\gamma$ and has a solution. Thus (3.21) has a solution if and only if (3.35) has a solution.

Since the value of $J^{*}(\beta)$ is determined by the existence of a solution to the Riccati equation (3.35), it is easy to see that there is a relationship between $J^{*}(\beta)$ and the interval length $T$. In particular, the larger $T$ is, the larger $J^{*}(\beta)$ is going to be.

The algorithm for determining $J^{*}(\beta)$ works as follows. Pick a $\gamma$, solve the Riccati equation (3.35) with a standard ode (ordinary differential equation) solver and see how far it goes. If it goes beyond $T$, then reduce $\gamma$ and start over. If the solution of the Riccati equation diverges before, increase $\gamma$ and start over. Using a simple bisection method, $J^{*}(\beta)$ can be found with desired accuracy.

Note however that for $\gamma=J^{*}(\beta)$, the Riccati equation (3.35) may diverge at $t^{*}<T$. In most cases, this does not happen. For example, it can be shown that if the models are time-invariant, the Riccati equation always diverges at $t=T$. To construct an academic example where $t^{*}<T$, consider the case where the matrix $C$ is identically zero over the interval $\left[t^{*}, T\right]$ for some $t^{*}<T$. If the Riccati equation (3.35) has a solution over $\left[0, t^{*}\right]$, then it has a solution over $[0, T]$ because this Riccati equation on $\left[t^{*}, T\right]$ is just a linear equation. So if $\gamma$ is such that (3.35) doesn't have a solution on $[0, T]$, it cannot have a solution on $\left[0, t^{*}\right]$ either.

To see the reason for this behavior, note that in this case, the observation of $y$ over $\left[t^{*}, T\right]$ does not provide any useful information for identification purposes. The separability index which is an increasing function of the length of the test period, is constant from $t^{*}$ on. In this case, there is no point using a test period with $T>t^{*}$ since we are not going to have a superior separability index anyway.

So, in the unlikely event that the Riccati equation (3.35) diverges at $t^{*}<T$, we simply reduce the test period by letting $T=t^{*}$. We will have then a faster identification scheme without having 
to pay a higher price in terms of the auxiliary signal energy. In the sequel, we shall suppose that the Riccati equation (3.35) diverges at $t=T$ and has a solution on $[0, T)$.

It should be noted that $\Pi(t)$ does not stay positive for all $t$. Typically, for $\gamma^{2}=J^{*}(\beta), \Pi(t)$ after starting off with initial condition $\Pi(0)=P_{\beta}>0$, becomes singular at some $t=t_{*}<T$. Beyond $t_{*}, \Pi(t)$ is no longer positive definite and as $t$ approaches $T$, at least one of its eigenvalues goes to minus infinity.

So when we pick a $\gamma$ and solve for $\Pi(t)$, for $\gamma$ large enough, say larger than some $\gamma_{h}(\beta)>0$, $\Pi(t)$ remains positive-definite on $[0, T]$. It can be shown that, in that case, the optimization problem

$$
\max _{v} \min _{(x, \nu)}\left\{L((x, \nu), \beta)-\gamma^{2} \int_{0}^{T}\|v\|^{2} d t\right\}
$$

has a saddle point. But this is not required here so $\gamma$ can be reduced. For $\gamma$ below $\gamma_{h}(\beta)$ but above $J^{*}(\beta), \Pi(t)$ becomes sign-indefinite but still does not diverge. And for $\gamma$ below $J^{*}(\beta), \Pi(t)$ diverges.

\subsection{Computation of the separability index $\gamma^{*}$}

In the previous section, we have seen how to compute $J^{*}(\beta)$, for any given $\beta$. Here, we use this to compute the separability index, based on (2.27). Even though $J^{*}(\beta)$ is not concave, it has nice properties making the optimization problem (2.27) numerically tractable. In particular, thanks to Lemma 2.2, we see that $J^{*}(\beta)$ is the supremum over concave functions each of which is zero at $\beta=0$ and $\beta=1$. Using this fact, we obtain the following result.

Lemma 3.7 For any $\beta_{1}$ and $\beta_{2}$ satisfying $0 \leq \beta_{1}<\beta_{2} \leq 1$,

$$
\max _{\beta_{1} \leq \beta \leq \beta_{2}} J^{*}(\beta) \leq \frac{J^{*}\left(\beta_{1}\right) J^{*}\left(\beta_{2}\right)}{J^{*}\left(\beta_{1}\right)\left(1-\beta_{2}\right)+J^{*}\left(\beta_{2}\right) \beta_{1}} .
$$

The proof follows a straightforward geometric argument illustrated in Figure 3.1. The key idea is that $J^{*}(\beta)$ is the supremum over concave functions going through the points $(0,0)$ and $(1,0)$, so it remains necessarily below the two dashed lines inside $\left[\beta_{1}, \beta_{2}\right]$ (and above outside).

Now consider the following simple optimization strategy for estimating $\gamma^{* 2}$ which consists of taking the maximum of $J^{*}(\beta)$ for $n$ regularly spaced values of $\beta$ over $[0,1]$ :

$$
\hat{\gamma}^{2}=\max _{k=1, \ldots, n} J^{*}(k /(n+1)) .
$$

Then, thanks to Lemma 3.7, it is straightforward to show that

$$
\left|\frac{\gamma^{* 2}-\hat{\gamma}^{2}}{\hat{\gamma}^{2}}\right| \leq \frac{1}{n+1}
$$

So for example by simply taking $\beta=1 / 2$, the worst possible error in $\gamma^{* 2}$ would be a factor of two.

The optimization method (3.37), thanks to (3.38), allows us to compute $\gamma^{*}$ with the desired precision. We can also use more sophisticated algorithms to estimate $\gamma^{*}$ and even couple the $\beta$ and $\gamma$ iterations.

RR $n^{\circ} 4000$ 


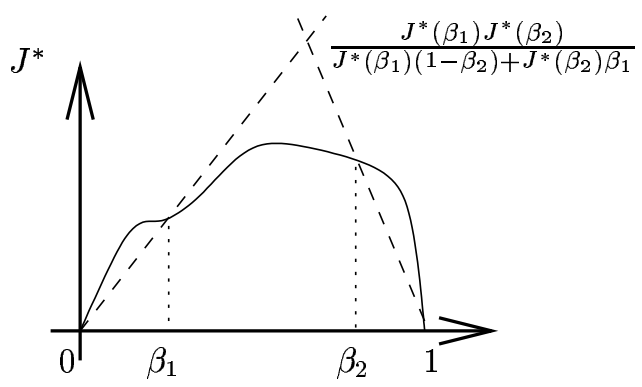

Figure 3.1: A typical $J^{*}(\beta)$ as a function of $\beta$.

\section{Construction of the auxiliary signal}

Once we have computed $\beta^{*}$, an optimal value of $\beta$ in (2.27), and the separability index $\gamma^{*}$, we can proceed with the construction of minimal energy proper auxiliary signal $v$.

\subsection{Characterization of the auxiliary signal}

As we have seen in Lemma 3.3, optimal $v$ can be found from any non-zero solution of the two-point boundary value system (3.17). This system depends on $P$, the solution of the Riccati equation (3.11), for $\beta=\beta^{*}$. We can simplify the system by removing this dependence as follows.

Lemma 4.1 Let

$$
x=\bar{x}+P \lambda .
$$

Then the two-point boundary value system (3.17) can be expressed as follows

$$
\frac{d}{d t}\left(\begin{array}{c}
x \\
\lambda
\end{array}\right)=\left(\begin{array}{cc}
A-S_{\beta^{*}} R_{\beta^{*}}^{-1} C & Q_{\beta^{*}}-S_{\beta^{*}} R_{\beta^{*}}^{-1} S_{\beta^{*}}^{T}-B B^{T} / \gamma^{* 2} \\
C^{T} R_{\beta^{*}}^{-1} C & -\left(A-S_{\beta^{*}} R_{\beta^{*}}^{-1} C\right)^{T}
\end{array}\right)\left(\begin{array}{l}
x \\
\lambda
\end{array}\right)
$$

with boundary conditions:

$$
\begin{aligned}
x(0) & =P_{\beta^{*}} \lambda(0) \\
\lambda(T) & =0 .
\end{aligned}
$$

We already know that this problem has non-zero solutions for $\beta^{*}$ and $\gamma^{*}$ that we have computed. It is exactly these solutions which give us the optimal auxiliary signal as

$$
v^{*}=\frac{B^{T} \lambda}{\gamma^{*} \sqrt{\int_{0}^{T}\left\|B^{T} \lambda\right\|^{2} d t}} .
$$

Note that the norm of $v^{*}$ equals $1 / \gamma^{*}$ giving $J_{v^{*}}\left(\beta^{*}\right)=1$ which is exactly the limit that guarantees that $v^{*}$ is proper (see (2.25)). 


\subsection{Computation of the auxiliary signal}

For the construction of the auxiliary signal, we need to solve the two-point boundary-value system (4.2).

Lemma 4.2 For any solution $(x, \lambda)$ of the boundary-value system (4.2), we have, over $[0, T)$,

$$
x=\Pi \lambda
$$

where $\Pi$ is given in (3.35) with $\beta=\beta^{*}$ and $\gamma=\gamma^{*}$.

Proof Suppose that $(x, \lambda)$ is a solution of (4.2)-(4.4). Let $\hat{\lambda}$ be the solution of

$$
\dot{\hat{\lambda}}=\left(C^{T} R_{\beta}^{-1} C \Pi-\left(A-S_{\beta^{*}} R_{\beta^{*}}^{-1} C\right)^{T}\right) \hat{\lambda}, \quad \hat{\lambda}(0)=\lambda(0) .
$$

Let $\hat{x}=\Pi \hat{\lambda}$. It is straightforward to verify that $(\hat{x}, \hat{\lambda})$ is a solution of (4.2)-(4.4). But $(x, \lambda)$ and $(\hat{x}, \hat{\lambda})$ are solutions of (4.2) which satisfy the same initial condition and hence $x=\hat{x}$.

We have seen that as $t$ goes to $T, \Pi(t)$ diverges so $\bar{\Pi}(T)=\lim _{t \rightarrow T} \Pi(t)^{-1}$ is singular. $\bar{\Pi}(T)$ can be computed by inverting $\Pi(t)$ for a $t$ close to $T$; this may not be numerically reliable.

The other way is that when we get close to $T$ (say at $t=T-\delta$ for some small $\delta$ ) and $\Pi(t)$ starts diverging, to switch from the Riccati equation (3.35) to the following equivalent Riccati equation where we have $\bar{\Pi}=\Pi^{-1}$

$$
\begin{aligned}
-\dot{\bar{\Pi}}=\bar{\Pi}\left(A-S_{\beta^{*}}\right. & \left.R_{\beta^{*}}^{-1} C\right)+\left(A-S_{\beta^{*}} R_{\beta^{*}}^{-1} C\right)^{T} \bar{\Pi}-C^{T} R_{\beta^{*}}^{-1} C+ \\
& \bar{\Pi}\left(Q_{\beta^{*}}-S_{\beta^{*}} R_{\beta^{*}}^{-1} S_{\beta^{*}}^{T}-B B^{T} / \gamma^{* 2}\right) \bar{\Pi}, \quad \bar{\Pi}(T-\delta)=\Pi^{-1}(T-\delta) .
\end{aligned}
$$

This equation integrates perfectly up to $T$ yielding a singular $\bar{\Pi}(T)$. The reason we do not use this Riccati equation from the beginning $(t=0)$ is that, as we have seen previously, $\Pi(t)$ becomes singular somewhere in the middle of the interval, which means that $\bar{\Pi}$ goes through infinity at that point. It is for this reason that $\delta$ must be chosen small enough to avoid this singularity, but not too small in order to avoid numerical problems with the inversion of $\Pi(T-\delta)$.

Once we have found $\bar{\Pi}(T)$, noting that

$$
\bar{\Pi}(T) x(T)=\lambda(T)=0,
$$

we can let $x(T)$ be any non zero vector in the null space of $\bar{\Pi}(T)$, say $x_{T} \neq 0$. We can now find a non zero solution to (4.2) by taking as boundary (final) condition

$$
x(T)=x_{T}, \lambda(T)=0 .
$$

This system is well-posed and has a unique solution. However, since this system is not backward stable, its numerical solution can result in large errors. The way to avoid this problem is to use (4.2) 
with boundary (final) condition (4.10) simply to solve for $(x, \lambda)$ over a short interval, for example $[T-\delta, T]$. Then from $T-\delta$ on, use

$$
\dot{\lambda}=\left(-A^{T}+C^{T} R_{\beta^{*}}^{-1} C \Pi+C^{T} R_{\beta^{*}}^{-1} S_{\beta^{*}}^{T}\right) \lambda
$$

to solve for $\lambda$ down to zero. This relation is obtained easily from (4.6). The optimal auxiliary signal is then given by (4.5).

\section{On-line identifi cation}

The computation of the auxiliary signal is done off-line, once and for all. The identification, which consists in deciding which model is the correct model, must be done on-line.

\subsection{Standard solution}

The standard solution to the on-line identification problem is to verify the compatibility of the observation $y$ with each of the two models. Let

$$
s_{i}(\tau)=\min \left\{x_{i}(0)^{T} P_{i, 0}^{-1} x_{i}(0)+\int_{0}^{\tau}\left\|\nu_{i}\right\|^{2} d t\right\} .
$$

subject to constraints $(2.1)$ and $(2.2)$ on $[0, \tau]$. Then clearly the observation $y$ is compatible with Model 0 if $s_{0}(T)<1$ and it is compatible with Model 1 if $s_{1}(T)<1$. If, as input, we use a proper auxiliary signal, we know that either $s_{0}(T) \geq 1$ or $s_{1}(T) \geq 1$. If $s_{0}(T)$ is larger than one, Model 1 is necessarily the correct model, if $s_{1}(T)$ is, then Model 0 is.

$s_{i}(\tau)$, for $0 \leq \tau \leq T$, are increasing functions and can be computed using standard Kalman filters. So we can implement the on-line identification procedure using two Kalman filters and threshold tests. The identification is made when $s_{0}(\tau)$ or $s_{1}(\tau)$ goes above one. In some cases, in particular when $\nu_{i}^{\prime} s$ don't take worst case values, this can happen even before the end of the time interval $(T)$.

The problem with this method however is that the on-line computation is intensive. Even if Kalman gain matrices are precomputed, the filter equations still require a lot of computation since the sizes of the states $x_{i}$ are usually quite large, especially compared to the size of $y$. The storage of the Kalman gain matrices also is not always possible because of their large sizes.

It is for these reasons that we seek a different method of identification, one that takes into account the fact that we know in advance the input signal (the auxiliary signal). The standard method does not take this into account.

\subsection{The hyperplane test}

The hyperplane test has been first introduced in [8]. Its application is based on the following result.

Lemma 5.1 Let $v$ be a proper auxiliary signal. Then there exists a hyperplane in $\mathcal{L}^{2}[0, T]$ separating the sets $\mathcal{A}^{0}(v)$ and $\mathcal{A}^{1}(v)$. 
Proof $\mathcal{A}^{0}(v)$ and $\mathcal{A}^{1}(v)$ are convex sets because they are images of convex sets, defined in (2.3), by linear operators (2.1)-(2.2). And, since $v$ is proper, we know that $\mathcal{A}^{0}(v)$ and $\mathcal{A}^{1}(v)$ are disjoint. So, the existence of the separating hyperplane is a direct consequence of the Hahn Banach Theorem (see in particular Corollary 1.1, Chapter I, of [3]).

The key point here is that the separating hyperplane does not depend on the observation $y$, so it can be computed off-line and implemented as follows

$$
\int_{0}^{T} h(t)^{T} y(t) d t \lesseqgtr a
$$

for some signal $h$ and scalar $a$. Note that both $h$ and $a$ are precomputed, and the size of $h$ equals that of $y$. So the complexity of this test is totally independent of the sizes of the $x_{i}$ 's.

The following Theorem gives a complete constructive method for designing a hyperplane test.

Theorem 5.1 Given the proper auxiliary signal (4.5) where $\lambda$ is a non-zero solution of the boundaryvalue system (4.2), for the hyperplane test (5.2), we can take

$$
h=R_{\beta^{*}}^{-1}\left(C \Pi+S_{\beta^{*}}^{T}\right) \lambda,
$$

where $\Pi$ is the solution of the Riccati equation (3.35) and

$$
a=\int_{0}^{T} h^{T} q d t
$$

where

$$
q=\left(\begin{array}{ll}
C_{0} & 0
\end{array}\right) \Pi \lambda+\left(\begin{array}{ll}
N_{0} & 0
\end{array}\right) V_{\beta^{*}}^{-1}\left(M^{T} \lambda-N^{T} h\right) .
$$

Proof Consider the boundary-value system (4.2) and in particular its solution corresponding to the choice (4.5). Denote this particular solution by $x^{*}$, and $\lambda^{*}$. Let

$$
\nu^{*}=V_{\beta^{*}}^{-1}\left(M^{T} \lambda^{*}-N^{T} \mu^{*}\right)
$$

where

$$
\mu^{*}=R_{\beta^{*}}^{-1}\left(C \Pi+S_{\beta^{*}}^{T}\right) \lambda^{*}
$$

We need now the following result.

Lemma $5.2\left(\left(x^{*}, \nu^{*}\right), \beta^{*}\right)$ is a saddle point of $L$ corresponding to $v=v^{*}$. 
Proof In view of Theorem 2.1, all we need to show is that $\left(x^{*}, \nu^{*}\right)$ is the solution of optimization problem (3.7) with $\beta=\beta^{*}$. The solution to this problem is completely characterized in terms of the necessary conditions obtained by the first order variation. Adding the constraints (3.2)-(3.3) to the cost function using lagrange multipliers $\sigma$ and $\omega$ and setting the first order variation to zero, we obtain the boundary-value system

$$
\begin{aligned}
\dot{x} & =A x+M \nu+B v^{*} \\
0 & =C x+N \nu \\
V_{\beta^{*}} & =M^{T} \sigma-N^{T} \omega \\
\sigma(0) & =P_{\beta^{*}}^{-1} x(0) \\
\sigma(T) & =0 .
\end{aligned}
$$

What we need to show is that there exist $\sigma$ and $\omega$ such that (5.8)-(5.12) are satisfied with $x=x^{*}$ and $\nu=\nu^{*}$.

From (4.2), we have that

$$
\begin{aligned}
x^{*} & =\left(A-S_{\beta^{*}} R_{\beta^{*}}^{-1} C\right) x^{*}+\left(Q_{\beta^{*}}-S_{\beta^{*}} R_{\beta^{*}}^{-1} S_{\beta^{*}}^{T}-B B^{T} / \gamma^{* 2}\right) \lambda^{*} \\
& =A x^{*}+\left(Q_{\beta^{*}}-S_{\beta^{*}} R_{\beta^{*}}^{-1}\left(C \Pi+S_{\beta^{*}}^{T}\right)\right) \lambda^{*}+B v^{*} \\
& =A x^{*}+Q_{\beta^{*}} \lambda^{*}+S_{\beta^{*}} \mu^{*}+B v^{*} \\
& =A x^{*}+M \nu^{*}+B v^{*} .
\end{aligned}
$$

This shows (5.8). We also have

$$
\begin{aligned}
C x^{*}+N \nu^{*} & =C \Pi \lambda^{*}+N V_{\beta^{*}}^{-1}\left(M^{T} \lambda^{*}-N^{T} \mu^{*}\right) \\
& =\left(C \Pi \lambda^{*}+S_{\beta^{*}}^{T}\right) \lambda^{*}-R_{\beta^{*}}^{-1} \mu^{*} \\
& =0 .
\end{aligned}
$$

This shows (5.9). The rest of the equations follow easily by setting $\sigma=\lambda^{*}$ and $\omega=\mu^{*}$.

Thus $x^{*}$ is the state trajectory of $x$ corresponding to $v^{*}$ and $\nu^{*}$. Let $x_{0}^{*}$ and $x_{1}^{*}$ denote the components of $x^{*}$ corresponding respectively to the states of model 0 and Model 1 . Similarly, define $\nu_{0}^{*}$ and $\nu_{1}^{*}$. Clearly the outputs corresponding to these time trajectories applied to the original systems (2.1)-(2.2), $i=0,1$, are identical; let's denote it by $y^{*}$. Since

$$
L\left(\left(x^{*}, \nu^{*}\right), \beta^{*}\right)=\min _{(x, \nu)} L\left((x, \nu), \beta^{*}\right)=J_{v^{*}}\left(\beta^{*}\right)=1,
$$

thanks to Corollary 2.1, we have

$$
\mathcal{S}_{0}\left(x_{0}^{*}(0), \nu_{0}^{*}\right)=\mathcal{S}_{1}\left(x_{1}^{*}(0), \nu_{1}^{*}\right)=1,
$$

which means that $y^{*}$ corresponds to a point on the intersection of the closures of $\mathcal{A}^{0}(v)$ and $\mathcal{A}^{1}(v)$.

Note that $\lambda^{*}$ is just a normalized version of $\lambda$. So, for some non zero scalar $c, \lambda=c \lambda^{*}$ and thus

$$
h=c \mu^{*}
$$


Now let us show that

$$
q=c y^{*} \text {. }
$$

For the computation of $y^{*}$, we can either use Model 0, or Model 1; we should obtain of course the same result. Using Model 0, we get

$$
\begin{aligned}
& y^{*}=C_{0} x_{0}^{*}+N_{0} \nu_{0}^{*} \\
& =\left(\begin{array}{ll}
C_{0} & 0
\end{array}\right) x^{*}+\left(\begin{array}{ll}
N_{0} & 0
\end{array}\right) \nu^{*} \\
& =\left(\begin{array}{ll}
C_{0} & 0
\end{array}\right) \Pi \lambda^{*}+\left(\begin{array}{ll}
N_{0} & 0
\end{array}\right) V_{\beta^{*}}^{-1}\left(M^{T} \lambda^{*}-N^{T} \mu^{*}\right) \\
& =\frac{1}{c} q
\end{aligned}
$$

which shows (5.18).

Now what we need to show is that for any $y$ in $\mathcal{A}^{0}(v),(5.2)$ is satisfied with the same inequality, and for any $y$ in $\mathcal{A}^{1}(v)$, it is satisfied with the opposite inequality.

Let $y$ be any output trajectory in $\mathcal{A}^{0}(v)$ (compatible with Model 0 ). This means that there exist $x_{0}(0)$ and $\nu_{0}$ satisfying

$$
\mathcal{S}_{0}\left(x_{0}(0), \nu_{0}\right)=x_{0}(0)^{T} P_{0,0}^{-1} x_{0}(0)+\int_{0}^{T}\left\|\nu_{0}\right\|^{2} d t<1
$$

for which $y$ is the output of (2.1)-(2.2), for $i=0$. What we need to show is that

$$
\int_{0}^{T} h^{T}(y-q) d t \quad \text { or equivalently } \quad \int_{0}^{T} \mu^{* T}\left(y-y^{*}\right) d t
$$

always has the same sign for any such $y$.

Using the relation

$$
\begin{aligned}
C^{T} \mu * & =C^{T} R_{\beta^{*}}^{-1}\left(C \Pi+S_{\beta^{*}}^{T}\right) \lambda^{*} \\
& =C^{T} R_{\beta^{*}}^{-1} C x^{*}+C^{T} R_{\beta^{*}}^{-1} S_{\beta^{*}}^{T} \lambda^{*} \\
& =-A^{T} \lambda^{*}-\dot{\lambda^{*}}
\end{aligned}
$$

$\mathrm{RR} \mathrm{n}^{\circ} 4000$ 
we obtain

$$
\begin{aligned}
\mu^{* T}\left(y-y^{*}\right)= & \mu^{* T}\left(C_{0}\left(x_{0}-x_{0}^{*}\right)+N_{0}\left(\nu_{0}-\nu_{0}^{*}\right)\right) \\
= & -\left({\dot{\lambda^{*}}}^{T}+\lambda^{* T} A_{0}\right)\left(\begin{array}{l}
I \\
0
\end{array}\right)\left(x_{0}-x_{0}^{*}\right)- \\
& \lambda^{* T}\left(\begin{array}{l}
I \\
0
\end{array}\right) M_{0}\left(\nu_{0}-\nu_{0}^{*}\right)-\beta^{*} \nu_{0}^{* T}\left(\nu_{0}-\nu_{0}^{*}\right) \\
= & -\left({\dot{\lambda^{*}}}^{T}+\lambda^{* T} A_{0}\right)\left(\begin{array}{l}
I \\
0
\end{array}\right)\left(x_{0}-x_{0}^{*}\right)-\lambda^{* T}\left(\begin{array}{l}
I \\
0
\end{array}\right)\left(\dot{x}_{0}-\dot{x}_{0}^{*}-\right. \\
& \left.A_{0}\left(x_{0}-x_{0}^{*}\right)\right)-\beta^{*} \nu_{0}^{* T}\left(\nu_{0}-\nu_{0}^{*}\right) \\
= & -{\dot{\lambda^{*}}}^{T}\left(\begin{array}{l}
I \\
0
\end{array}\right)\left(x_{0}-x_{0}^{*}\right)-\lambda^{* T}\left(\begin{array}{l}
I \\
0
\end{array}\right)\left(\dot{x}_{0}-\dot{x}_{0}^{*}\right)- \\
& \beta^{*} \nu_{0}^{* T}\left(\nu_{0}-\nu_{0}^{*}\right)
\end{aligned}
$$

Integrating the result from 0 to $T$, using integration by parts, the fact that $\lambda^{*}(T)=0$, (4.6) and (5.16), we get

$$
\begin{aligned}
\int_{0}^{T} \mu^{* T}\left(y-y^{*}\right) d t= & -x^{*}(0) \Pi^{-1}(0)\left(\begin{array}{l}
I \\
0
\end{array}\right)\left(x_{0}(0)-x_{0}^{*}(0)\right)- \\
& \beta^{*} \int_{0}^{T} \nu_{0}^{* T}\left(\nu_{0}-\nu_{0}^{*}\right) d t \\
= & -\beta^{*} x_{0}^{*}(0) P_{0,0}\left(x_{0}(0)-x_{0}^{*}(0)\right)- \\
& \beta^{*} \int_{0}^{T} \nu_{0}^{* T}\left(\nu_{0}-\nu_{0}^{*}\right) d t \\
= & \beta^{*}\left(1-x_{0}^{*}(0) P_{0,0} x_{0}(0)+\int_{0}^{T} \nu_{0}^{* T} \nu_{0} d t\right)
\end{aligned}
$$

which is positive thanks to (5.23), (5.16) and the Schwarz inequality. This shows that for all $y$ in $\mathcal{A}^{0}(v)$,

$$
\int_{0}^{T} \mu^{* T}\left(y-y^{*}\right)>0 .
$$

Similarly, we can show that for all $y$ in $\mathcal{A}^{1}(v)$,

$$
\int_{0}^{T} \mu^{* T}\left(y-y^{*}\right)<0 .
$$

This completes this proof. 


\section{Example and discussion}

Programs implementing the methodology presented in this paper can easily be developed in software environments such as Scilab [1] and Matlab. For the computation of the separability index, the main function to have is one that given a pair $(\beta, \gamma)$, determines whether or not the Riccati equation (3.35) has a solution. This is easily implemented by a call to an ode solver. The separability index can be computed by a series of calls to this function as explained in previous sections ( $\gamma$ and $\beta$ iterations).

The computation of the optimal proper auxiliary signal and the associated hyperplane parameters does not require an iterative procedure. Assuming $\beta^{*}$ and $\gamma^{*}$ are already computed, it requires only a nontrivial solution to the boundary value problem (4.2). The only (minor) difficulty is that for numerical stability, it is better to store the solution of the Riccati equation (3.35) corresponding to $\beta^{*}$ and $\gamma^{*}$ and use it to integrate $\lambda$ backwards as we have seen in Section 4.

The method presented in this paper allows us to deal with large systems without much difficulty. Here we present an example which has been treated in Scilab on a PC. The computation takes a few minutes (depending on the desired accuracy on the computation of $\gamma^{*}$ ).

In this example, we use two randomly selected time-invariant models where $v$ has size 2 and $y$, size 3. The sizes of $x_{0}, x_{1}, \nu_{0}$ and $\nu_{1}$ are respectively $8,5,4$ and 4 . The test period is [0,7].

The optimal auxiliary signal is illustrated in Figure 6.1. The hyperplane test in this case can be based on $h(t)$ in Figure 6.2.

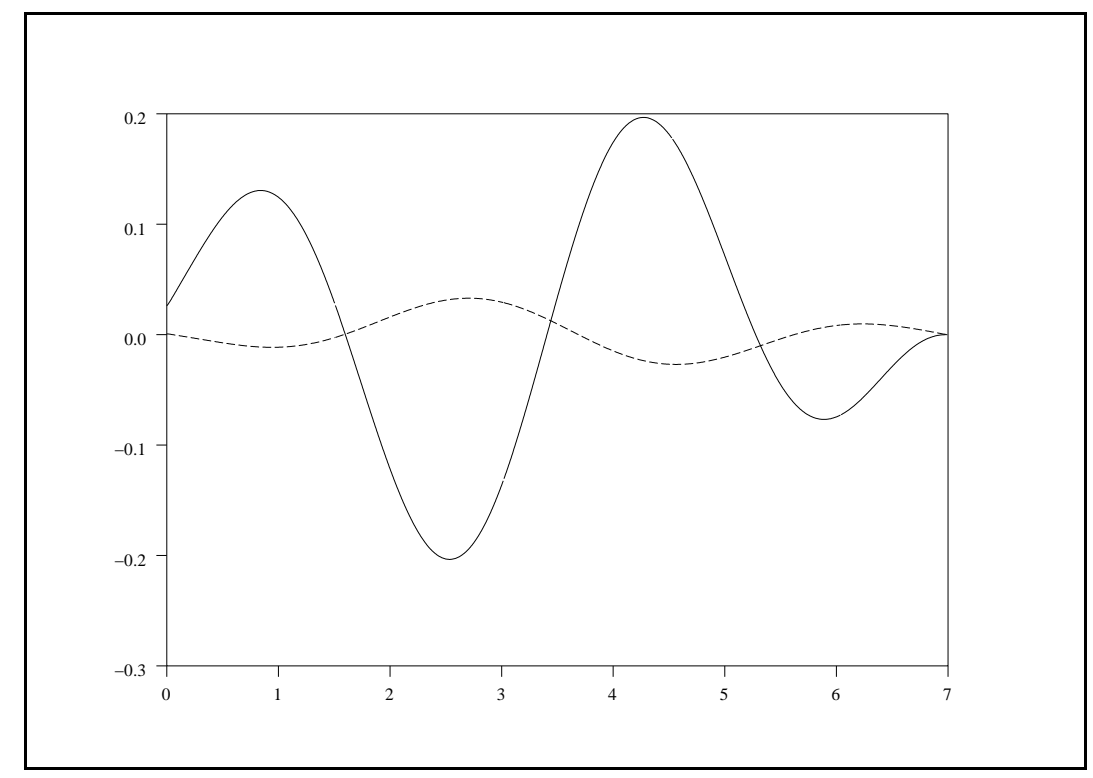

Figure 6.1: The two entries of the optimal auxiliary signal. 


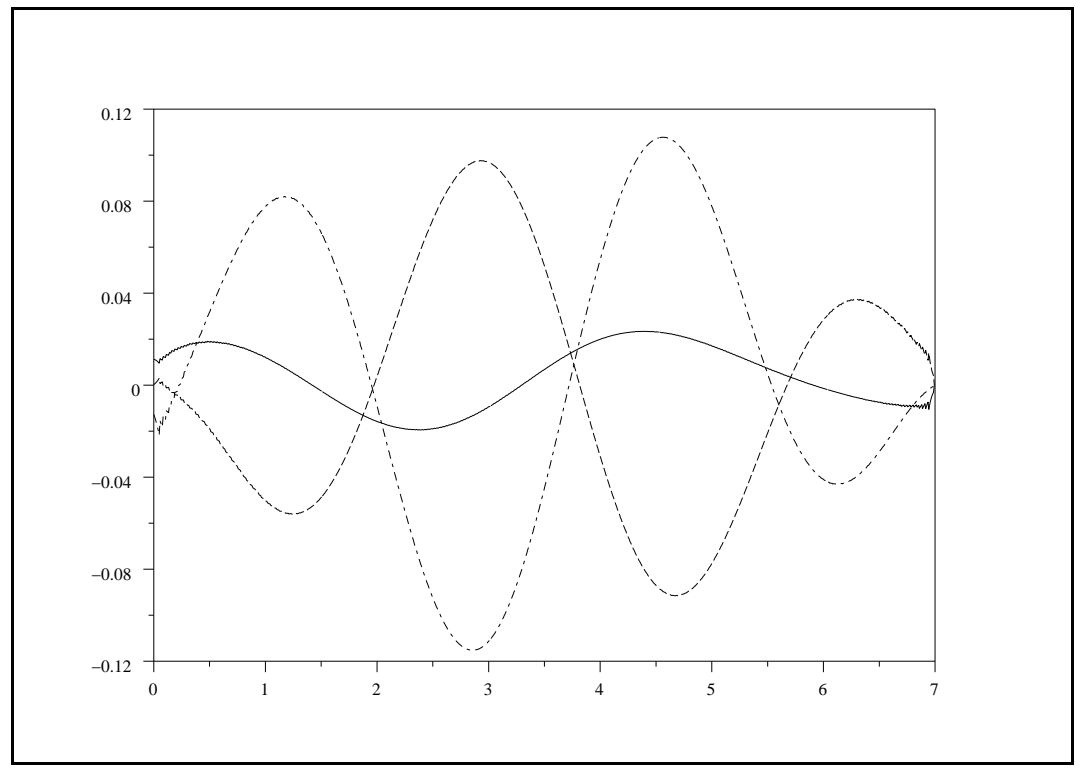

Figure 6.2: The three entries of $h(t)$.

The signals $v^{*}$ and $h$ appear to have a dominant frequency. The same thing is observed on other examples and becomes even clearer when we increase the length of the test period. This is not a surprise because, assuming the two candidate models are time-invariant, as we let $T$ go to infinity, the solution of the optimization problem $J^{*}\left(\beta^{*}\right)$ becomes the $H_{\infty}$ norm of the system (3.12)-(3.13), and $v^{*}$ tends to a pure sinusoid whose frequency corresponds to maximum gain of the norm of the transfer function associated with this system. This point has been discussed in [9].

If the two models are stable and satisfy the following condition

$$
\left(\begin{array}{cc}
s I-A_{i} & M_{i} \\
C_{i} & N_{i}
\end{array}\right) \quad \text { has full row rank } \forall s \text { on the } j \omega \text {-axis. }
$$

then, as $T$ goes to infinity, $\gamma^{*}=J^{*}\left(\beta^{*}\right)$ can be computed as the infimum over the set of $\gamma^{\text {'s for }}$ which the Hamiltonian matrix

$$
\left(\begin{array}{cc}
A-S_{\beta^{*}} R_{\beta^{*}}^{-1} C & Q_{\beta^{*}}-S_{\beta^{*}} R_{\beta^{*}}^{-1} S_{\beta^{*}}^{T}-B B^{T} / \gamma^{2} \\
C^{T} R_{\beta^{*}}^{-1} C & -\left(A-S_{\beta^{*}} R_{\beta^{*}}^{-1} C\right)^{T}
\end{array}\right)
$$

has no eigenmodes on the $j \omega$-axis. This means that it can be obtained directly without having to solve any Riccati equation. For $\gamma=\gamma^{*}$, the Hamiltonian does have an eigenmode $j \omega^{*}$ on the $j \omega$-axis. It is the value of this $\omega^{*}$ which determines the frequency of $v^{*}$. 
Note that the infinite horizon problem is not of direct interest because it does not make sense to have a test period of infinite length. It does however provide a better comprehension of the mathematical problem and can be used to develop efficient coding methods for $v^{*}$ and $h$, and for approximation techniques when the test interval is long (as done in [9]).

In general of course, the objective is to have a short test interval. But there is a trade-off between the separability index and the length of the test period. As we have seen, $\gamma^{*}$ is an increasing function of $T$.

\section{Conclusions}

We have presented a methodology for error-free system identification in the situation where we have two candidate linear models subject to bounded energy noise, and where we have control over the input. The problem of selecting a best input signal over a test period (the minimum proper auxiliary signal design problem) has been solved and a solution given in terms of the solution to a boundary value system.

The solution of this boundary value system also provides us with means to design a very efficient on-line identification scheme (the hyperplane test); one that takes into account the fact that the input signal over the test period is known in advance.

A related problem which can be solved with the methodology presented here is the shortest test period problem where we fix the separability index and look for the shortest test period for perfect identification. The procedure is similar to the one presented in this paper, simply the $\gamma$-iteration part should be replaced with $T$-iteration. This is particularly useful when we have more than two candidate models. Suppose we have three: Model 0, Model 1 and Model 2. In that case, one way to approach this problem is to apply first an auxiliary signal to separate say Model 0 from Model 1. Then, depending on the result of the first test, apply another test to separate Model 2 from Model 0 or from Model 1. In general, $N-1$ tests are required for $N$ candidate models if this approach is used.

There are a number of possible extensions to the work presented here. There is of course the extension to discrete time. This is straightforward.

The other extension is to allow for additional inputs to the system, i.e., the models would have another input $u$ (in addition to the auxiliary signal $v$ ), but this one measured on-line, just like the output $y$. This situation has been considered in [9]. The idea is to eliminate $u$ just as we eliminated $y$ to obtain (2.10). This leads to a descriptor system (3.2)-(3.3). In [9], a method was presented for reducing the descriptor system to a smaller, explicit system. A similar method can be used in the continuous-time case, even if the models are time varying. There are however some technical issues that arise: for example the $\mathcal{A}^{i}(v)$ are not necessarily bounded. But the separating hyperplane does exist and can be constructed with similar methods.

Another possible extension to this work is to allow for some nonlinearity. In particular, if the system is not linear in $v$, the Kalman filter implementation and the hyperplane test still apply. The problem is the optimization over $v$. An approach using optimization software is discussed in [2] which may be able to deal with such systems. 
Finally, there is the possibility of allowing $v$ to depend on the measured $y$. This problem, which we call the closed-loop auxiliary signal design problem, will be presented in a future paper. Unfortunately in this case the hyperplane test cannot be used anymore (because $v$ is not know in advance), but using the additional information contained in the output $y$ can improve the performance of the on-line identification scheme.

\section{References}

[1] Bunks, C., Chancelier, J.P., Delebecque, F., Gomez, C. (Editor), Goursat, M., Nikoukhah, R. and Steer, S., Engineering and scientific computing with Scilab, Birkhauser, 1999.

[2] Campbell, S.L., Horton, K., Nikoukhah, R. and Delebecque, F., Rapid model selection and the separability index, Proc. 4th IFAC Symposium on Fault Detection Supervision and Safety for Technical Processes (SAFEPROCESS 2000), Budapest, Hungary, 1187-1192.

[3] Ekeland, I., and Temam, R., Convex analysis and variational problems, SIAM, 1999.

[4] Gelb, A., Applied Optimal Estimation, M.I.T. Press, 1984.

[5] Kerestecioğlu, F., Change detection and input design in dynamical systems, Research Studies Press, Taunton, U.K., 1993.

[6] Kerestecioğlu, F. and Zarrop, M. B., Input design for detection of abrupt changes in dynamical systems, Int. J. Control, vol. 59, no. 4, 1994, pp. 1063-1084.

[7] Kullback, S., Information Theory and Statistics, John Wiley \& Sons, 1971.

[8] Nikoukhah, R., Guaranteed active failure detection and isolation for linear dynamical systems, Automatica, vol. 34, no. 11, 1998.

[9] Nikoukhah, R., Campbell, S. L. and Delebecque, F., detection signal design for failure detection: a robust approach, Internat. J. Adaptive Control and Signal Processing, vol. 14, 2000.

[10] Petersen, I.R., Savkin, A.V., Robust Kalman filtering for signals and systems with large uncertainties, Birkhauser, 1999.

[11] Uosaki, K, Tanaka, I., and Sugiyama, H., Optimal input design for autoregressive model discrimination with constrained output variance, IEEE Trans. Auto. Contr., vol. AC-29, no. 4, 1984, pp. 348-350.

[12] Zhang, X. J., Auxiliary signal design in fault detection and diagnosis, Springer-Verlag, Heidelberg, 1989. 


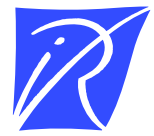

Unité de recherche INRIA Rocquencourt Domaine de Voluceau - Rocquencourt - BP 105 - 78153 Le Chesnay Cedex (France)

Unité de recherche INRIA Lorraine : LORIA, Technopôle de Nancy-Brabois - Campus scientifi que 615, rue du Jardin Botanique - BP 101 - 54602 Villers-lès-Nancy Cedex (France)

Unité de recherche INRIA Rennes : IRISA, Campus universitaire de Beaulieu - 35042 Rennes Cedex (France)

Unité de recherche INRIA Rhône-Alpes : 655, avenue de l'Europe - 38330 Montbonnot-St-Martin (France)

Unité de recherche INRIA Sophia Antipolis : 2004, route des Lucioles - BP 93 - 06902 Sophia Antipolis Cedex (France)

INRIA - Domaine de Voluceau - Rocquencourt, BP 105 - 78153 Le Chesnay Cedex (France)

http://www.inria.fr

ISSN 0249-6399 